\title{
LIMIT THEOREMS FOR CONTINUOUS STATE BRANCHING PROCESSES WITH IMMIGRATION
}

\author{
BY MARK A. PINSKY ${ }^{1}$
}

Communicated by Harry Kesten, November 4, 1971

Introduction. K. Kawazu and S. Watanabe [5] have defined a CBI process as a Markov process $X=\left(x_{t}, P_{x}\right)$ with state space $[0, \infty]$, with $\infty$ as a trap, possessing the property that, for each $t \geqq 0, \lambda \geqq 0$, there exist $\varphi(t, \lambda) \geqq 0$ and $\psi(t, \lambda) \geqq 0$ such that

$$
E_{x}\left[e^{-\lambda x_{t}} ; t<e_{\infty}\right]=\varphi(t, \lambda) e^{-x \psi(t, \lambda)},
$$

for every $x \in[0, \infty]$; here $e_{\infty}=\inf \left\{t: x_{t}=\infty\right\}$. Previously Lamperti [6] had treated the case $\varphi \equiv 1$.

The Markov property of $X$ implies that, for $\lambda \geqq 0, s, t \geqq 0$,

$$
\begin{aligned}
& \psi(t+s, \lambda)=\psi(t, \psi(s, \lambda)), \\
& \varphi(t+s, \lambda)=\varphi(t, \lambda) \varphi(s, \psi(t, \lambda)) .
\end{aligned}
$$

Under the condition of right continuity of $X$ at $t=0$, it follows from (1.2) and (1.3) that $\psi$ and $\varphi$ are differentiable. Explicitly, we have

$$
\begin{aligned}
\partial \psi / \partial t & =R(\psi), \quad \psi\left(0^{+}, \lambda\right)=\lambda, \\
\varphi(t, \lambda) & =\exp \left(-\int_{0}^{t} F(\psi(s, \lambda)) d s\right)
\end{aligned}
$$

for appropriate functions $R$ and $F$. Kawazu and Watanabe have used the property (1.1) to show that they must have the form

$$
\begin{aligned}
& R(\lambda)=-\alpha \lambda^{2}+\beta \lambda+\gamma-\int_{0^{+}}^{\infty}\left(e^{-\lambda x}-1+\frac{\lambda x}{1+x^{2}}\right) n_{1}(d x), \\
& F(\lambda)=c+d \lambda-\int_{0^{+}}^{\infty}\left(e^{-\lambda x}-1\right) n_{2}(d x),
\end{aligned}
$$

when $n_{1}$ and $n_{2}$ are measures on the Borel sets of $(0, \infty)$ with the property that

$$
\int_{0^{+}}^{\infty} \frac{u^{2}}{1+u^{2}} n_{1}(d u)+\int_{0^{+}}^{\infty} \frac{u}{1+u} n_{2}(d u)<\infty ; \quad \alpha \geqq 0, \gamma \geqq 0, c \geqq 0, d \geqq 0 .
$$

Furthermore any set of parameters $\left(\alpha, \beta, \gamma, c, d, n_{1}, n_{2}\right)$ define a unique

AMS 1970 subject classifications. Primary 60J80, 60F05; Secondary 60K30.

Key words and phrases. Branching processes, limiting distributions, nonlinear normalizations, linear dam model.

${ }^{1}$ Supported by NSF Grant GP-9437 and GP-28576. 
CBI process. In this note we shall only deal with conservative processes. According to Kawazu and Watanabe, this is equivalent to $\gamma=c=0$ and $\int_{0^{+}}^{\infty} R^{*}(\lambda)^{-1} d \lambda=+\infty$ where $R^{*}(\lambda)=\max (R(\lambda), 0)$. This is satisfied, for instance, in the case $\int_{0}^{\infty} x n_{1}(d x)<\infty$, which we shall explicitly assume; let

$$
\rho=R^{\prime}(0)=\beta-\int_{0}^{\infty} \frac{x^{3}}{1+x^{2}} n_{1}(d x) .
$$

We shall first give a general result and then proceed to examine special cases.

\section{Statement of results.}

THEOREM. Let $X=\left(x_{t}, P_{x}\right)$ be a conservative CBI process with $\int_{1}^{\infty} x n_{1}(d x)$ $<\infty$. Let $\rho(t)=e^{\rho t}$ if $\rho>0 ; \rho(t)=1$ if $\rho \leqq 0$. As $t \rightarrow \infty, x_{t} / \rho(t)$ converges in distribution to a proper random variable if and only if

$$
\int_{0}^{1} \frac{F(\lambda)}{|R(\lambda)|} d \lambda<\infty
$$

Corollary 1. Let $\rho>0, \int_{1}^{\infty} x \log x n_{1}(d x)<\infty$. Then as $t \rightarrow \infty, x_{t} / e^{\rho t}$ has a proper, nondegenerate limiting distribution if and only if

$$
\int_{1}^{\infty}(\log x) n_{2}(d x)<\infty
$$

The convergence takes place almost surely and in $L^{1}$ mean.

CoROLlary 2. Let $\rho<0$. Then as $t \rightarrow \infty, x_{t}$ has a proper, nondegenerate limiting distribution if and only if (B) is satisfied.

Corollary 3. Let $\rho=0, \int_{1}^{\infty} x^{2} n_{1}(d x)=\infty$. Then as $t \rightarrow \infty, x_{t}$ has a proper, nondegenerate limiting distribution if and only if $(\mathrm{A})$ is satisfied.

For comparison with known theorems for Galton-Watson processes we give the following result which applies in the case of finite variance.

Corollary 4. Let $\rho=0, \int_{1}^{\infty} x^{2} n_{1}(d x)<\infty, \int_{1}^{\infty} x n_{2}(d x)<\infty$; then as $t \rightarrow \infty, x_{t} / t$ has a proper, nondegenerate limiting distribution.

A short calculation shows that the condition (B) is a special case of the condition (A) for the case $R(\lambda)=\lambda$. This case appeared [1] in the study of a storage system proposed by Moran [7]. Condition (B) has appeared in the study of discrete parameter, discrete state branching processes, by Heathcote [3], [4]. Corollary 3 is related to a result of Seneta [8]. Recent work of Foster and Williamson [2] extend Seneta's observations.

When condition (B) fails, the following result gives a nonlinear normalization which produces weak convergence. We know of no analogue in the 
discrete parameter case. For simplicity, we state the result in the subcritical case.

THEOREM 2. Let $X=\left(x_{t}, P_{x}\right)$ be a conservative CBI process with $-\infty<\rho$ $<0$. For $x>0$ let

$$
H(x)=\int_{e^{-x}}^{1} \frac{F(u)}{R(u)} d u, \quad m(x)=\exp (H(\log x)) .
$$

Assume that as $x \rightarrow \infty$, we have

$$
\begin{aligned}
H(x) & \rightarrow \infty, \\
x H^{\prime}(x) & \rightarrow 0 .
\end{aligned}
$$

Then for $0 \leqq u \leqq 1$,

$$
P_{x}\left\{m\left(x_{t}\right) / m\left(e^{c t}\right) \leqq u\right\} \rightarrow u^{1 / c},
$$

as $t \rightarrow \infty$, here $c=-\rho<0$.

This result covers cases in which the integral (B) diverges "slowly." Condition (C2) holds, for example, if $H(x)=\log \log x$; then $\left(\log \log x_{t}\right) /(\log c t)$ converges weakly to a limit. If $H(x)=\log x$, condition (C2) fails; a direct calculation shows nonetheless that we have $\left(\log x_{t}\right) / c t$ weakly convergent when $t \rightarrow \infty$. If $H(x)=x^{1 / 2}$, a direct calculation shows that, as $t \rightarrow \infty$, $\left(\log x_{t}\right) / t^{2}$ converges weakly; this is not of the above form (*).

Professor Michael B. Marcus has made the useful observation that $H(x)$ can be expressed directly in terms of the distribution $n_{2}$ by the relation $H(x) \sim$ const $\int_{1}^{x}\left(n_{2}([u, \infty) / u) d u\right)$.

It is a pleasure to thank Dr. K. B. Erickson for several helpful conversations relative to the above work.

\section{REFERENCES}

1. E. Cinlar and M. Pinsky, A stochastic integral in storage theory, Z. Wahrscheinlichkeitstheorie und Verw. Gebiete 17 (1971), 227-240.

2. J. Foster and J. A. Williamson, Limit theorems for the Galton-Watson process with time-dependent immigration, Z. Wahrscheinlichkeitstheorie und Verw. Gebiete 20 (1971), 227-235.

3. C. R. Heathcote, A branching process allowing immigration, J. Roy, Statist. Soc. Ser. B 27 (1965), 138-143. MR 33 \#1896a.

4. , Correction to above paper, J. Roy. Statist. Soc. Ser. B 28 (1966), 213-217. MR 33 \# 1896b.

5. K. Kawazu and S. Watanabe, Branching processes with immigration and related limit theorems, Teor. Verojatnost i Primenen. 16 (1971), 34-51.

6. J. Lamperti, Continuous state branching processes, Bull. Amer. Math. Soc. 73 (1967), 382-386. MR 34 \#8494.

7. P. A. P. Moran, $A$ theory of dams with continuous input and a general release rule, J. Appl. Probability 6 (1969), 88-98. MR 39 \# 2232.

8. E. Seneta, The stationary distributions of a branching process allowing immigration: A remark on the critical case, J. Roy. Statist. Soc. Ser. B 30 (1968), 176-179. MR 37 \# 4888. 9. - Functional equations and the Galton-Watson process, Advances in Appl. Probability 1 (1969), 1-42. MR 40 \#2167.

DePartment of MATHEMATICS, NORThWestern University, Evanston, Illinois 60201 